\title{
Operational Characteristics of SiC Diodes as Ionizing Radiation Detectors
}

\section{De Napoli ${ }^{1}$}

INFN - Laboratori Nazionali del Sud

Via S. Sofia 62, 95123 Catania-Italy

E-mail: denapolielns.infn.it

F. Giacoppo

Dipartimento di Fisica, Università degli Studi di Messina

Via Salita Sperone 31, I-98166 Messina-Italy

\section{G. Raciti}

Dipartimento di Fisica e Astronomia, Università degli Studi di Catania Via S. Sofia 64, 95123 Catania-Italy

\section{E. Rapisarda}

INFN - Sezione di Catania

Via S. Sofia 64, 95123 Catania-Italy

\section{Sfienti}

Dipartimento di Fisica e Astronomia, Università degli Studi di Catania Via S. Sofia 64, 95123 Catania-Italy

\begin{abstract}
The operational characteristics of $4 \mathrm{H}-\mathrm{SiC}$ Schottky diodes with different dopant concentrations have been studied with ${ }^{12} \mathrm{C}$ and ${ }^{16} \mathrm{O}$ ions at various incident energies. The detector response has been investigated in term of linearity, energy resolution, signal rise-time and Charge Collection Efficiency as a function of the applied reverse bias and of the dopant concentration. Moreover, since one of the most promising properties of $\mathrm{SiC}$ detectors is their radiation hardness, the radiation damage, produced by irradiating $\mathrm{SiC}$ diodes with ${ }^{16} \mathrm{O}$ ions at $35.2 \mathrm{MeV}$, has been evaluated by measuring the degradation of both the signal pulse-height and the energy resolution as a function of the ${ }^{16} \mathrm{O}$ fluence.
\end{abstract}

XLVIII International Winter Meeting on Nuclear Physics - BORMIO2010

Bormio, Italy

January 25-29 2

\footnotetext{
1 Speaker
} 


\section{Introduction}

Silicon Carbide $(\mathrm{SiC})$ is a wide bandgap compound semiconductor existing in many different polytypes, depending on the difference in the stacking of the Si-C pair. $\mathrm{SiC}$ crystals have several interesting characteristics as a wide bandgap, a high electron saturation velocity, a large dielectric breakdown voltage, a good thermal conductivity and a predicted radiation hardness [1]. Therefore $\mathrm{SiC}$ detectors are presently developed for use in high-temperature and high radiation conditions under which conventional semiconductor detectors cannot adequately perform [2]. In spite of the notable development in growing, processing and producing good-quality and low-defect $\mathrm{SiC}$ diodes, the characterization of such devices as radiation detectors is far from completed. We have therefore studied the response signal and the radiation damage of high-quality $4 \mathrm{H}-\mathrm{SiC}$ Schottky diode with different doping concentrations by using ${ }^{12} \mathrm{C}$ and ${ }^{16} \mathrm{O}$ ions at various incident energies [3-5].

\section{Experimental Details}

Three types of SiC Schottky diodes (named A,B,C), with different $\mathrm{n}^{-}$epitaxial layer concentrations $\left(7.6 \times 10^{14}, 2.0 \times 10^{15}, 1.5 \times 10^{16} \mathrm{~N} / \mathrm{cm}^{3}\right)$ and thicknesses $(37.9,43.7$, $21.0 \mu \mathrm{m}$ ), have been used. The active area of each chip is $2 \times 2 \mathrm{~mm}^{2}$, with capacitances ranging from about 5 and $16 \mathrm{pF}$. The dopant nitrogen concentration and thickness of the $\mathrm{n}^{+}$side are $7 \times 10^{18} \mathrm{~N} / \mathrm{cm}^{3}$ and $279 \mu \mathrm{m}$, respectively (Fig. 1 left panel). The diodes have been fabricated by epitaxy onto high-purity $4 \mathrm{H}-\mathrm{SiC}$ n-type substrate from the ETCCatania [6] by using different wafers. The chips were glued onto a brass foil $1 \mathrm{~mm}$ thick by conductive glue and single contacts between the $\mathrm{Ni}_{2} \mathrm{Si}$ front surfaces and individual pads of a board were realized by Al wire ( $2 \mu \mathrm{m}$ thick) bonding (Fig. 1 right panel).

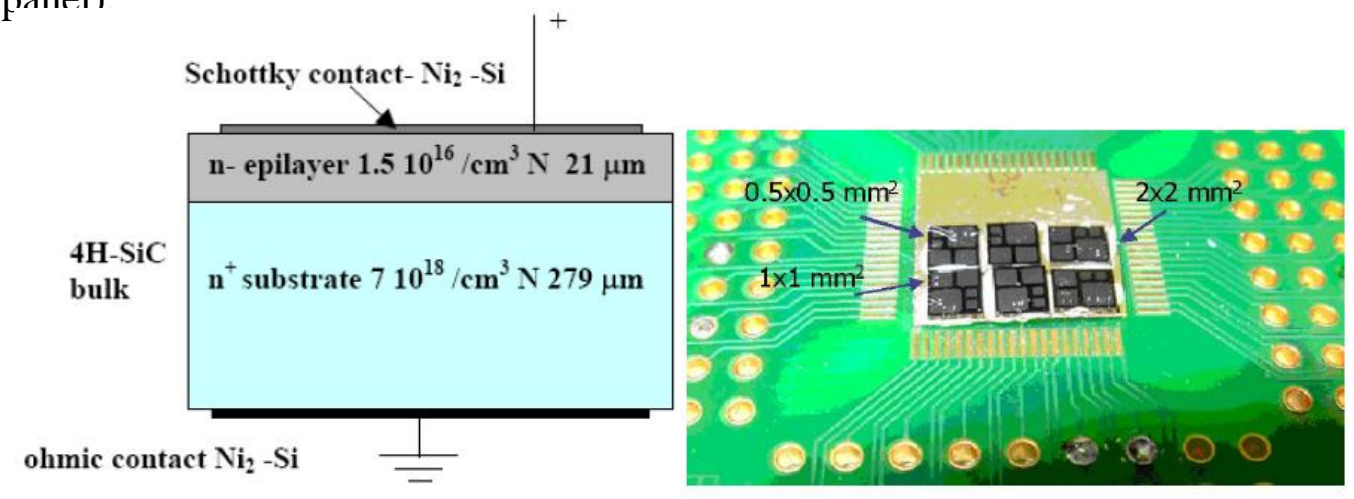

Figure 1. Left panel: Type c diode layout. Right panel: Picture of the SiC detectors assembled on a board.

The boards were set-up in a scattering chamber at the Laboratori Nazionali del Sud (LNS-Catania) and operated under vacuum at $10^{-6} \mathrm{mbar}$. Standard electronics were used to process the signals: preamplifiers of $45 \mathrm{mV} / \mathrm{MeV}$ gain (ORTEC 142A) and amplifiers with $0.5 \mu \mathrm{sec}$ shaping time. 


\section{Detector Response}

The three types of $\mathrm{SiC}$ diodes were used to detect ${ }^{12} \mathrm{C}$ ions at $13.7,27.7$ and 37.3 $\mathrm{MeV}$. For each incident energy, a number of energy spectra were taken for different values of reverse bias. The pulse-height peak position moves towards higher channel values as the voltage increases until it reaches the saturation when the bias depletes the active volume of the diode up to the range of the incoming ${ }^{12} \mathrm{C}$ ions. The range values the three ${ }^{12} \mathrm{C}$ incident energies are 8.5, 19.7 and $29.1 \mu \mathrm{m}$, respectively. In Fig. 2 (left panel) the correlation between the square-root of the applied bias and the depleted thickness is shown. The experimental data are compared with predictions from [7]. As expected, $\mathrm{SiC}$ diodes with lower dopant concentration require lower reverse bias values to deplete the same thickness [4]. Arrows in Fig. 2 (left panel) indicate the bias needed to fully deplete the diodes. These values are 805, 2322 and 2062 Volts for SiC type a, b, and c, respectively.
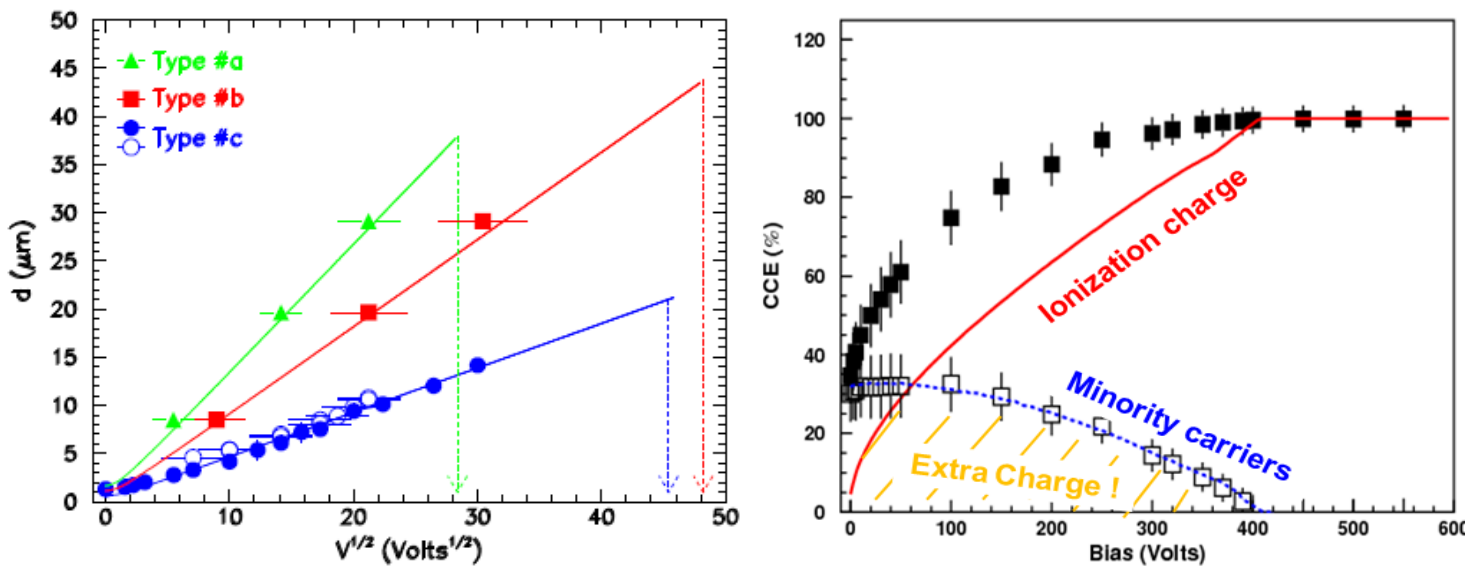

Figure 2. Left-panel: correlation between the square-root of the applied bias and the depletion layer thickness of $\mathrm{SiC}$ a (triangles), b (squares) and c (full and empty circles) [4]. The lines are theoretical predictions from [7]. Arrows indicate the bias needed to fully deplete the diodes. Right-panel: CCE of a SiC type b (full squares) as a function of the applied reverse bias for ${ }^{12} \mathrm{C}$ ions at $27.7 \mathrm{MeV}$ [5]. The full curve represents the theoretical contribution from ionization charges. The curve saturation is reached when the bias depletes the active volume of the diode up to the range of the incoming ${ }^{12} \mathrm{C}$ ions. The empty squares are the differences between the experimental points and the full curve. The dashed curve represent the contribution from minority carriers [8].

All the tested detectors show a high degree of linearity between the saturated pulse-height value and the deposited energy $[3,4]$. The measured energy resolution ranges from $5.5 \%$ down to $1.7 \%$ [4] and the signal rise-time from 40 to $100 \mathrm{nsec}$ [3]. The influence of the used preamplifier to the measured signal rise-time was less than 6 nsec. Finally, Fig. 2 right-panel shows the Charge Collection Efficiency (CCE), defined as the ratio between the collected charge and the charge produced by the particle in the detector, as a function of the applied bias [5]. The amount of the collected charge is proportional to the peak position. In particular, since in the signal saturation region all the produced charge is collected, the total charge produced by ${ }^{12} \mathrm{C}$ in the $\mathrm{SiC}$ diode is 
proportional to the saturated peak position. The experimental CCE is therefore extracted as the ratio between the peak position, measured at different applied bias, and its saturation value. In the bias region where the CCE is not saturated, the calculations of CCE due to ionization charges underestimate the experimental data. This result indicates that, in this bias region, more charge than the ionization charge produced by the ions in the depleted layer is collected. There is a good agreement between the extra CCE (empty points) and the Breese predictions (dashed curve) [8]. Therefore the extra collected charge is due to the minority carriers, generated by the incoming ion in the neutral region of the diode, which diffuse to the depleted layer and are finally collected $[5]$.

\section{Radiation Damage}

One of the most interesting properties of $\mathrm{SiC}$ detectors is their radiation hardness. An important task of this work was to explore the effects of a reduced dopant concentration in the radiation hardness properties of $\mathrm{SiC}$ detectors. SiC of type a and $\mathrm{c}$ were irradiated using ${ }^{16} \mathrm{O}$ ions at $35.2 \mathrm{MeV}[3,4]$. During the irradiation the reverse bias of $\mathrm{SiC}$ a and c was kept fixed at a value of 600 and 400 Volts, corresponding to a depletion thickness of 32.7 and $9.2 \mu \mathrm{m}$, respectively. The ratio between the peak centroid of the ${ }^{16} \mathrm{O}$ energy spectrum after the irradiation $\left(\mathrm{PC}_{A I}\right)$ over the same peak centroid before the irradiation $\left(\mathrm{PC}_{B I}\right)$, is shown in Fig. 3 (left panel) as a function of the ${ }^{16} \mathrm{O}$ fluence. It is evident that, by increasing the fluence, the energy peak, for both $\mathrm{SiC} \mathrm{a}$ (triangles) and c (full and empty circles), moves toward lower channels, indicating an increasing incompleteness in the charge collection. In particular, the signal amplitude of $\mathrm{SiC}$ type c drops to $50 \%$ at a fluence of $6.5 \times 10^{14} \mathrm{ions} / \mathrm{cm}^{2}$, whereas the one of SiC type a drops to $50 \%$ already at a fluence of $4.1 \times 10^{14}$ ions $/ \mathrm{cm}^{2}$ (see lines in Fig. 3). Therefore the diode with a factor 20 lower dopant concentration shows a radiation hardness reduced by $60 \%$. When defects are formed inside the $\mathrm{SiC}$ lattice, the energy resolution of the detector is worsen due to fluctuations in the amount of charge lost. Figure 8 (right panel) shows the relation between the relative energy resolution and the ${ }^{16} \mathrm{O}$ fluence for $\mathrm{SiC} \mathrm{a}$ (triangles) and c (circles). The energy resolution of $\mathrm{SiC}$ a degrades more than the one of $\mathrm{SiC} \mathrm{c}$ for increasing fluence. Indeed, the relative energy resolution of $\mathrm{SiC} \mathrm{c}$ gets ten times worse at a fluence of $7.7 \times 10^{14}$ ions $/ \mathrm{cm}^{2}$, whereas the one of $\mathrm{SiC}$ a worsen by the same amount already for $2.8 \times 10^{14}$ ions $/ \mathrm{cm}^{2}$ (see lines in Fig. 3 right panel).

\section{Conclusions}

The response of $4 \mathrm{H}-\mathrm{SiC}$ Schottky diodes with different dopant concentration to ${ }^{12} \mathrm{C}$ and ${ }^{16} \mathrm{O}$ low energetic ions has been studied. The reverse bias voltages needed to deplete the same active volume of $\mathrm{SiC}$ diodes decrease at decreasing dopant concentration. Moreover, the signal response to the ionization produced by the lowenergy ions was analyzed in terms of linearity, energy resolution, rise-time and CCE. For the three $\mathrm{SiC}$ types, the experimental CCE increases by increasing the reverse bias, since the diode active layer widens and more energy is deposited within the depleted thickness. When the whole ${ }^{12} \mathrm{C}$ incident energy is deposited within the depleted thickness the CCE is $100 \%$. In the other cases the calculations of CCE due to ionization charges underestimate the experimental data. This result indicates that more charge than 
the ionization charge produced by the ions in the depleted layer is collected. The extra collected charge is due to the minority carriers, generated by the incoming ion in the neutral region of the diode, which diffuse to the depleted layer and are finally collected.
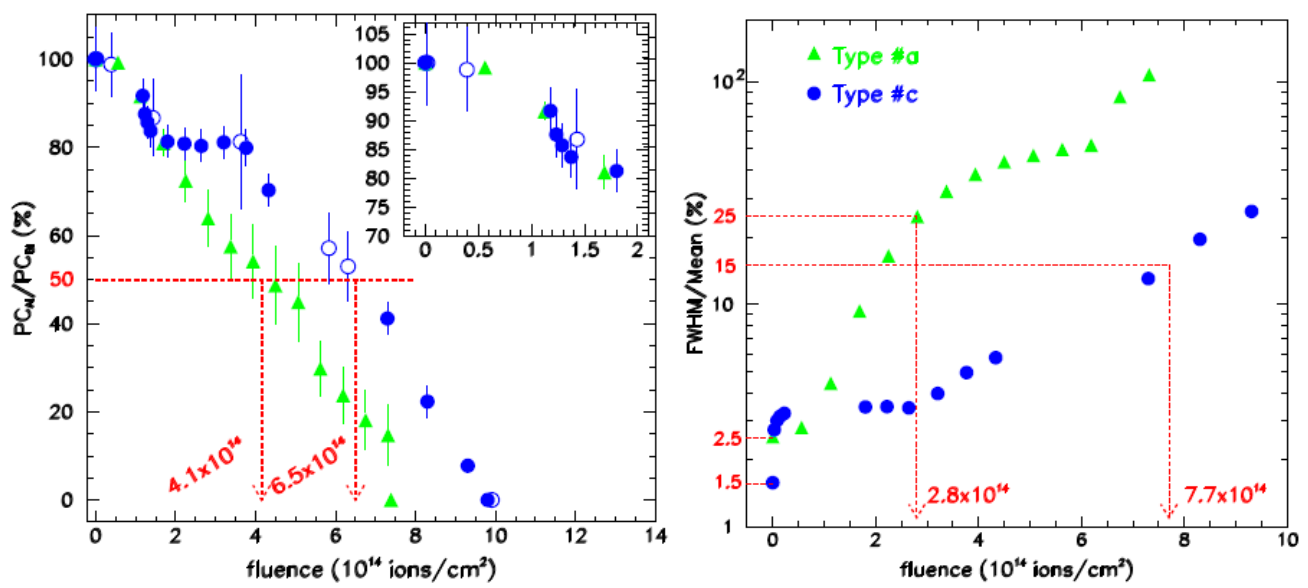

Figure 8. Left panel: Ratio of the peak centroid channel of the ${ }^{16} \mathrm{O}$ energy spectrum after the irradiation $\left(\mathrm{PC}_{\mathrm{AI}}\right)$ and before the irradiation $\left(\mathrm{PC}_{\mathrm{BI}}\right)$ at increasing fluence for $\mathrm{SiC}$ type a (triangles) and c (circles) [4]. Right panel: Relative energy resolution as a function of the fluence [4].

Finally, the diodes have been irradiated with ${ }^{16} \mathrm{O}$ ions at $35.2 \mathrm{MeV}$ and the radiation hardness properties were studied by measuring the degradation of the signal as a function of the ${ }^{16} \mathrm{O}$ fluence. In case of $\mathrm{SiC}$ type $\mathrm{c}$ the amplitude of the signal drops down to $50 \%$ at a fluence of $6.5 \times 10^{14}$ ions $/ \mathrm{cm}^{2}$ indicating the good quality of the $\mathrm{SiC}$ as a radiation hard material. However, the $\mathrm{SiC}$ diode with lower dopant concentration, suffered a faster reduction of the signal height and a larger deterioration of the energy resolution. A systematic collection of other experimental data is mandatory to assess the role of the dopant concentration and of other possible intrinsic properties of the single chip, in the radiation hardness properties of SiC detectors.

\section{References}

1. M. Iwami, Nucl. Instr. and Meth. A 466, (2001) 406.

2. F. Nava, G. Bertuccio, A. Cavallini, E. Vittone, Meas. Sci. Technol. 19, (2008) 102001.

3. M. De Napoli, G. Raciti, E.Rapisarda, C. Sfienti, Nucl. Instr. and Meth. A 572, (2007) 831.

4. M. De Napoli, F. Giacoppo, G.Raciti, E.Rapisarda, Nucl. Instr. and Meth. A 600, (2009) 618.

5. M. De Napoli, F. Giacoppo, G.Raciti, E.Rapisarda, Nucl. Instr. and Meth. A 608, (2009) 80.

6. E.T.C. Epitaxial Tecnology Center 207, Corso Italia I-95127 Catania -Italy.

7. G.F. Knoll, Radiation Detection and Measurement, Third Edition,Wiley, New York, 2000, p.393.

8. M.B.H. Breese, J. Appl. Phys. 74, (1993) 3789. 